\title{
METAIS PESADOS EM SOLOS DE ÁREA DE MINERAÇÃo E METALURGIA DE CHUMBO. II - FORMAS E DISPONIBILIDADE PARA PLANTAS ${ }^{(1)}$
}

\author{
Maurício Gomes de Andrade ${ }^{(2)}$, Vander de Freitas Melo(3), Luiz \\ Cláudio de Paula Souza ${ }^{(4)}$, Juarez Gabardo ${ }^{(5)}$ \& Carlos Bruno \\ Reissmann $^{(4)}$
}

\begin{abstract}
RESUMO
As formas e a disponibilidade dos metais pesados em solos contaminados definem o potencial de absorção pelas plantas e de contaminação das águas por lixiviação. Neste trabalho, foram usados diferentes métodos de extrações químicas com o objetivo de identificar as formas de $\mathrm{Pb}, \mathrm{Cd}, \mathrm{Cr}, \mathrm{Cu}, \mathrm{Ni}$ e $\mathrm{Zn}$ e avaliar a disponibilidade desses poluentes para girassol (Helianthus annuus L.), aveia-preta (Avena strigosa Schreber) (exóticas) e grama-batatais (Paspalum notatum Flügge) (nativa) como espécies indicadoras em solos de área de mineração e metalurgia de $\mathrm{Pb}$, no município de Adrianópolis (PR). Foram coletadas amostras (0 a $40 \mathrm{~cm}$ ) em quatro ambientes da área: solo 1 - referência (mata nativa); solo 2 pequena ocorrência de rejeitos no perfil; solo 3 - próximo da chaminé da fábrica (aporte de material particulado); solo 4 - intenso descarte de resíduos finos. Os métodos de extração empregados foram: DTPA-TEA pH 7,3; $\mathrm{Ca}\left(\mathrm{NO}_{3}\right)_{2} \mathbf{0 , 5} \mathrm{mol} \mathrm{L}^{-1}$; $\mathrm{HNO}_{3} 0,5,1,0$ e 4,0 $\mathrm{mol} \mathrm{L}^{-1}$ é água régia $\left(\mathrm{HNO}_{3} / \mathrm{HCl}\right.$ concentrados - 3:1). O experimento foi realizado em casa de vegetação, com três repetições. Houve intenso incremento nos teores pseudototais, trocáveis e não trocáveis de metais pesados com a mineração; os teores máximos de $\mathrm{Pb}$ e $\mathrm{Zn}$ extraídos pela água régia foram de 9.678,2 e 894,8 $\mathrm{mg} \mathrm{kg}^{-1}$, respectivamente. Os extratores $\mathrm{HNO}_{3} 0,5,1,0$ e 4,0 mol L-1 e água régia correlacionaram entre si quanto à extração de metais pesados nos solos. $O$
\end{abstract}

\footnotetext{
(1) Parte do trabalho de mestrado do primeiro autor apresentado ao Programa de Pós-Graduação em Ciência do Solo, Universidade Federal do Paraná. Recebido para publicação em março de 2009 e aprovado em outubro de 2009.

(2) Professor do Departamento de Química e Biologia, Universidade Tecnológica Federal do Paraná - UTFPR. Av. Sete de Setembro 3165, CEP 80203-901 Curitiba (PR). E-mail: mandrade@utfpr.edu.br

(3) Professor do Departamento de Solos e Engenharia Agrícola, Universidade Federal do Paraná - UFPR. Rua dos Funcionários 1540, Juvevê, CEP 80035-050 Curitiba (PR). Bolsista CNPq. E-mail: vanderfm@ufpr.br

(4) Professor do Departamento de Solos e Engenharia Agrícola, UFPR. E-mails: lcsouza@ufpr.br; reissman@ufpr.br

(5) Professor do Departamento de Genética, Universidade Federal do Paraná - UFPR. Centro Politécnico, Jardim das Américas, CEP 81531-990 Curitiba (PR). E-mail: jgabardo@ufpr.br
} 


\begin{abstract}
extrator com princípio de quelação (DTPA-TEA pH 7,3) não foi eficiente na predição da disponibilidade desses metais para as plantas. As extrações nítricas devem ser preferidas para se estabelecer a fitodisponibilidade de $\mathrm{Pb}$ e $\mathrm{Zn}$ nos solos da área.
\end{abstract}

Termos de indexação: extratores de metais pesados, girassol, aveia-preta, gramabatatais.

\title{
SUMMARY: HEAVY METALS IN SOILS OF A LEAD MINING AND METALLURGY AREA. II - FORMS AND PLANT AVAILABILITY
}

\begin{abstract}
The forms and availability of heavy metals in contaminated soils determine the uptake potential of plants and water contamination by leaching. In this study different methods of chemical extraction were used, in order to identify the forms of $\mathrm{Pb}, \mathrm{Cd}, \mathrm{Cr}, \mathrm{Ni}, \mathrm{Cu}$, and $\mathrm{Zn}$ and evaluate the availability of these pollutants for sunflower (Helianthus annuus L.), black oat (Avena strigosa Schreber) (exotic species) and Bahiagrass (Paspalum notatum Flügge) (native species), as indicators, in soils of Pb mining and metallurgy in Adrianópolis, state of Paraná, Brazil. Soil samples were collected in the 0-40 cm layer in four environments of the area: soil 1 - control (native forest); soil 2 - waste in soil profile; soil 3 - near the factory chimney (deposition of particulate material); soil 4 - heavy deposition of waste. The following extraction methods were used: DTPA-TEA pH 7.3; $\mathrm{Ca}\left(\mathrm{NO}_{3}\right)_{2} 0.5 \mathrm{~mol} \mathrm{~L}^{-1} ; \mathrm{HNO}_{3}$ 0.5, 1.0 and $4.0 \mathrm{~mol} \mathrm{~L}$, and concentrated $\mathrm{HNO}_{3} / \mathrm{HCl}$ (3:1). The plants were grown in a greenhouse, with three replications. The increase of pseudo-total, exchangeable and non-exchangeable heavy metal contents was verified; the highest contents of $\mathrm{Pb}$ and $\mathrm{Zn}$ were extracted by $\mathrm{HNO}_{3} / \mathrm{HCl} ; 9,678.2$ and $894.8 \mathrm{mg} \mathrm{kg}^{-1}$, respectively. Soil heavy metals based on acid extractors $\mathrm{HNO}_{3}$ 0.5, 1.0 and $4.0 \mathrm{~mol} \mathrm{~L}^{-1}$ and $\mathrm{HNO}_{3} / \mathrm{HCl}$ were strongly correlated with each other. The extractor based on chelation (DTPA-TEA pH 7.3) was not efficient to predict the availability of heavy metals for plants under the study conditions. The nitric extractions should be preferred to determine $\mathrm{Pb}$ and $Z n$ plant availability in the area.
\end{abstract}

Index terms: heavy metal extractors, sunflower, black oat, Bahiagrass.

\section{INTRODUÇÃO}

A alteração dos teores naturais dos metais pesados nos solos pela aplicação de fertilizantes, pulverização com agroquímicos, incorporação de biossólidos, atividades de mineração e metalurgia, entre outros, provoca uma demanda de informações sobre a adequação de extratores químicos para previsão de sua disponibilidade para plantas e outros organismos e para avaliar a mobilidade e o risco de poluição das águas subterrâneas.

As espécies de metais pesados nos solos podem ser definidas "funcionalmente" (disponíveis às plantas, formas móveis, trocáveis, etc.) e "operacionalmente" (tipo de procedimento de determinação e de reagentes ou extratores) (Ure et al., 1993). Os metais pesados podem ser encontrados no solo nas seguintes formas (Alloway, 1995): (a) solução do solo; (b) adsorvidos eletrostaticamente aos sítios de troca (adsorção não específica); (c) incorporados na superfície da fase inorgânica, como óxidos de $\mathrm{Al}, \mathrm{Fe}$ e Mn (adsorção específica); (d) participando de reações de precipitação e dissolução; (e) ligados a compostos orgânicos.
Muitos estudos foram realizados sobre a eficiência dos extratores para avaliar a disponibilidade de metais pesados, porém ainda não foi estabelecido um método universal que estime eficientemente os teores fitodisponíveis em diferentes solos e para diferentes espécies de plantas (Milagres et al., 2007). Soluções de agentes complexantes, principalmente DTPA, têm sido empregadas para extração de metais pesados trocáveis e associados preferencialmente à matéria orgânica, em solos tratados ou não com biossólidos (Borges \& Coutinho, 2004; Pierangeli et al., 2007). Para avaliação de formas mais disponíveis (trocáveis), é comum o emprego de soluções de sais neutros, como o $\mathrm{Ca}\left(\mathrm{NO}_{3}\right)_{2}$ 0,5 mol L-1 (Miller et al., 1986). Outros estudos envolvem métodos de extrações mais agressivas, por meio de soluções ácidas mais concentradas (Campos et al., 2005; Melo et al., 2006), como a água régia $\left(\mathrm{HNO}_{3} / \mathrm{HCl}\right.$ concentrados - 3:1) (Nieuwenhuize et al., 1991; USEPA, 2007). Essa extração visa determinar o potencial de disponibilidade e de mobilidade dos metais pesados nos solos (Rauret, 1998). Soluções ácidas mais diluídas são usadas para estimar os teores não trocáveis e de disponibilidade de metais pesados a curto/médio prazos. $\mathrm{O} \mathrm{HNO}_{3}$ 
$1 \mathrm{~mol} \mathrm{~L}^{-1}$ é empregado para avaliar as formas fortemente adsorvidas e mais uma parte da fração estrutural (Schalscha et al., 1980; Soon \& Bates, 1982).

Neste trabalho, foram usados diferentes métodos de extrações químicas com os objetivos de identificar as formas de $\mathrm{Pb}, \mathrm{Cd}, \mathrm{Cr}, \mathrm{Cu}, \mathrm{Ni}$ e $\mathrm{Zn}$ e avaliar a disponibilidade desses elementos para o girassol (Helianthus annuus L.), aveia-preta (Avena strigosa Schreber) e grama-batatais (Paspalum notatum Flügge) em solos de área de mineração e metalurgia de $\mathrm{Pb}$ em Adrianópolis (PR).

\section{MATERIAL E MÉTODOS}

\section{Área de estudo e amostragem dos solos}

A área de mineração e metalurgia de $\mathrm{Pb}$ situa-se no município de Adrianópolis (PR), na localidade de Panelas de Brejaúvas, distante $12 \mathrm{~km}$ da cidade e próximo das margens do rio Ribeira, fronteira com o Estado de São Paulo. Em 1995, após 50 anos de atividade, uma empresa mineradora encerrou suas atividades deixando a céu aberto, sem nenhuma proteção, aproximadamente $177 \mathrm{Gg}$ de resíduos de beneficiamento de $\mathrm{Pb}$ (Cassiano, 2001).

Os critérios para seleção dos solos foram apresentados por Andrade et al. (2009). As características dos locais são apresentadas no quadro 1 .
Foram coletados aproximadamente $100 \mathrm{~kg}$ de solo na profundidade de 0 a $40 \mathrm{~cm}$ em cada local selecionado. Para a instalação do experimento em casa de vegetação, as amostras foram secas ao ar e passadas em peneira de plástico de malha de $5 \mathrm{~mm}$. Aproximadamente $0,5 \mathrm{~kg}$ de solo seco ao ar foi passado em peneira de $2 \mathrm{~mm}$ (terra fina seca ao ar - TFSA) para a determinação de diferentes formas de $\mathrm{Pb}, \mathrm{Cd}$, $\mathrm{Cr}, \mathrm{Cu}, \mathrm{Ni}$ e $\mathrm{Zn}$.

\section{Teores pseudototais}

A digestão das amostras de solo em triplicata foram realizadas em forno de microondas, marca Milestone, modelo ETHOS 1, segundo o método SW 846-3051A (USEPA, 2007). Aproximadamente 0,5 g de solo foi digerido com $9 \mathrm{~mL}$ de ácido nítrico (65\%) e $3 \mathrm{~mL}$ de ácido clorídrico (36 \%) (água régia), em pré-aquecimento por $5 \mathrm{~min}$, a uma potência de $1.000 \mathrm{~W}$, até atingir a temperatura de $175 \pm 5{ }^{\circ} \mathrm{C}$, mantida por 10 min. Em seguida, a amostra foi resfriada por cerca $30 \mathrm{~min}$, mantida no interior do aparelho. Todo o filtrado (solução da extração mais da lavagem dos frascos) foi coletado em balão volumétrico de $50 \mathrm{~mL}$ e o volume completado com água deionizada. Determinaram-se os teores de $\mathrm{Pb}$, $\mathrm{Cd}, \mathrm{Cr}, \mathrm{Cu}, \mathrm{Ni}$ e $\mathrm{Zn}$ por espectroscopia de emissão atômica com plasma indutivamente acoplado (ICPAES) em aparelho Perkin Elmer, modelo Optima $3300 \mathrm{DV}$.

Quadro 1. Características dos locais de amostragem identificadas em campo

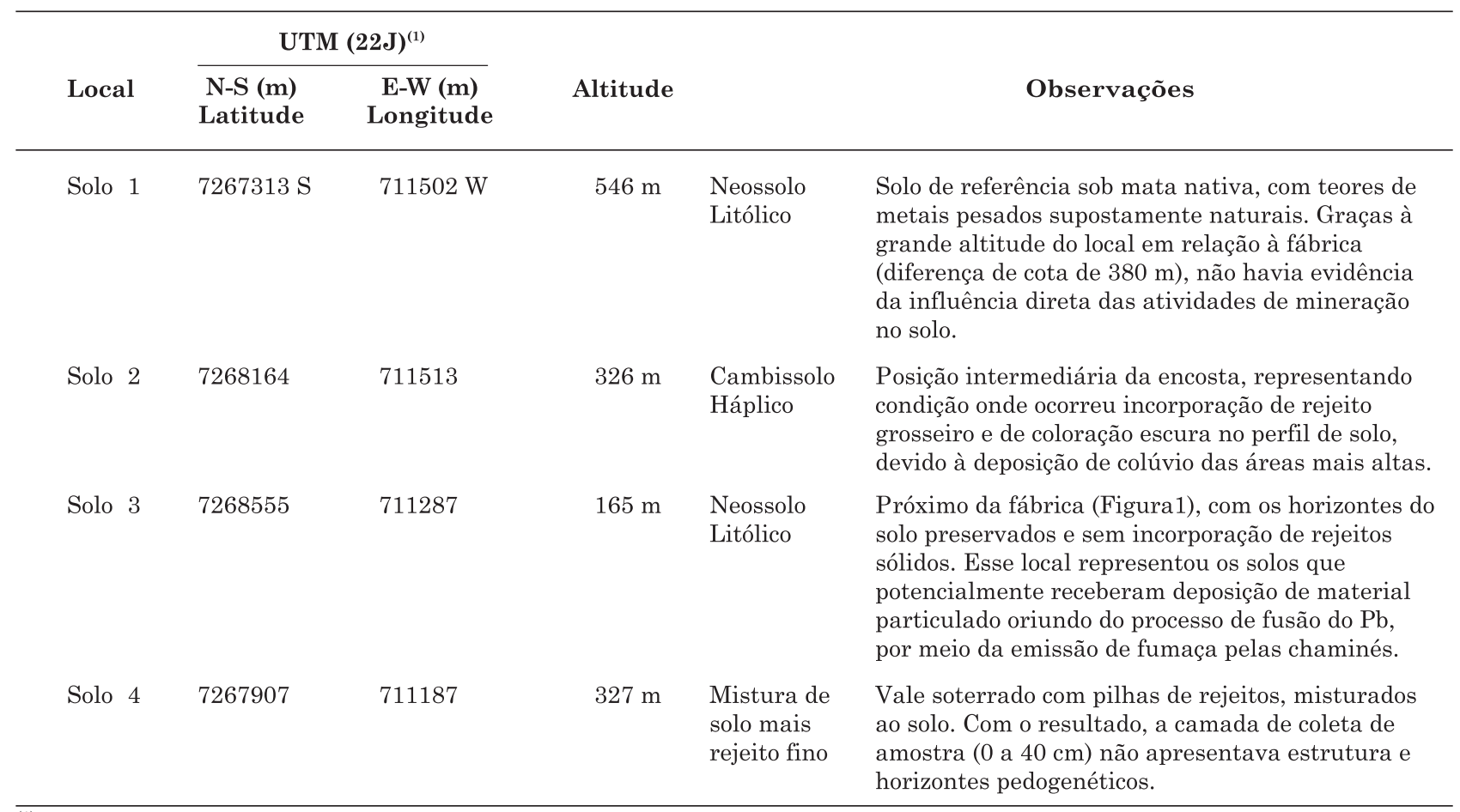

(1) Coordenadas planas do sistema UTM. 


\section{Teores trocáveis}

$$
\mathrm{Ca}\left(\mathrm{NO}_{3}\right)_{2} \cdot 4 \mathrm{H}_{2} \mathrm{O} 0,5 \mathrm{~mol} \mathrm{~L}^{-1}
$$

Transferiram-se cerca de $2 \mathrm{~g}$ de TFSA para erlenmeyer de $125 \mathrm{~mL}$, adicionaram-se $20 \mathrm{~mL}$ de solução de $\mathrm{Ca}\left(\mathrm{NO}_{3}\right)_{2} \cdot 4 \mathrm{H}_{2} \mathrm{O} 0,5 \mathrm{~mol} \mathrm{~L}^{-1}$ e agitou-se a suspensão por $1 \mathrm{~h}$, de acordo com Miller et al. (1986). A suspensão foi filtrada e os teores de $\mathrm{Pb}, \mathrm{Cd}, \mathrm{Cr}, \mathrm{Cu}$, $\mathrm{Ni}$ e Zn foram determinados por ICP-AES.

\section{Ácido dietilenotriaminopentacético e trietanolamina - DTPA-TEA}

Em erlenmeyer de $125 \mathrm{~mL}$, colocaram-se $10 \mathrm{~g}$ de TFSA e adicionaram-se $20 \mathrm{~mL}$ de solução de ácido dietilenotriaminopentacético (DTPA) 0,005 $\mathrm{mol} \mathrm{L}^{-1}+$ trietanolamina (TEA) $0,1 \mathrm{~mol} \mathrm{~L}^{-1}+\mathrm{CaCl}_{2} 0,01 \mathrm{~mol} \mathrm{~L}^{-1}$ em pH 7,3 (Lindsay \& Norvell, 1978). A suspensão foi agitada por $2 \mathrm{~h}$, em $220 \mathrm{rpm}$, e filtrada. Os teores de $\mathrm{Pb}, \mathrm{Cd}, \mathrm{Cr}, \mathrm{Cu}, \mathrm{Ni}$ e $\mathrm{Zn}$ foram determinados por ICP-AES.

\section{Teores não trocáveis}

Para a extração de metais pesados não trocáveis, foi utilizada solução de $\mathrm{HNO}_{3}$ fervente (Knudsen et al., 1986) em diferentes concentrações: 0,$5 ; 1,0$ e $4,0 \mathrm{~mol} \mathrm{~L}^{-1}$.

Cerca de $10 \mathrm{~g}$ de solo foram colocados para reagir com $25 \mathrm{~mL}$ de solução de $\mathrm{HNO}_{3}$ durante 25 min em banho de óleo vegetal sobre chapa de aquecimento, em temperatura de $113^{\circ} \mathrm{C}$. Em seguida, a suspensão foi filtrada e o resíduo lavado quatro vezes com $15 \mathrm{~mL}$ de solução de ácido nítrico 10 vezes menos concentrada em relação à solução de extração $\left(0,05 ; 0,1 ; 0,4 \mathrm{~mol} \mathrm{~L}^{-1}\right)$. Todo o filtrado (extração mais lavagem) foi recolhido em um balão volumétrico de $100 \mathrm{~mL}$ e o volume completado com a mesma solução de lavagem. Os teores de $\mathrm{Pb}, \mathrm{Cd}, \mathrm{Cr}, \mathrm{Cu}, \mathrm{Ni}$ e $\mathrm{Zn}$ foram analisados por ICP-AES.

\section{Experimento em casa de vegetação}

Foram utilizadas as espécies Avena strigosa Schreber (aveia-preta), Paspalum notatum Flügge (grama-batatais) e Helianthus annuus L. (girassol), e o experimento foi realizado em casa de vegetação, em vasos com $2 \mathrm{dm}^{3}$ de solo, com três repetições e em blocos ao acaso. Os vasos foram irrigados com água deionizada a $85 \%$ da capacidade de campo.

Após 71 dias da semeadura (início da fase reprodutiva do girassol e da aveia-preta) e do plantio dos estolões de grama-batatais, as plantas foram coletadas e separadas em parte aérea e raízes. As amostras foram lavadas, secas a $60^{\circ} \mathrm{C}$ até massa constante, e moídas para a determinação dos teores de metais pesados no tecido vegetal (raiz e parte aérea) por digestão úmida em sistema aberto com a mistura $\mathrm{HNO}_{3}$ e $\mathrm{HClO}_{4}$ (Hunter, 1975). Os teores de $\mathrm{Pb}, \mathrm{Cd}$, $\mathrm{Cr}, \mathrm{Cu}, \mathrm{Ni}$ e $\mathrm{Zn}$ foram analisados por ICP-AES.
Detalhes da instalação e realização do experimento em casa de vegetação e os resultados de matéria seca e teores de metais nas plantas foram apresentados por Andrade et al. (2009).

O solo de cada unidade experimental foi seco ao ar e passado em peneira de malha $2 \mathrm{~mm}$ (TFSA) para a determinação dos teores trocáveis de $\mathrm{Pb}, \mathrm{Cd}, \mathrm{Cr}, \mathrm{Cu}$, $\mathrm{Ni}$ e Zn com $\mathrm{Ca}\left(\mathrm{NO}_{3}\right)_{2} \cdot 4 \mathrm{H}_{2} \mathrm{O} 0,5 \mathrm{~mol} \mathrm{~L}^{-1}$ e solução de DTPA-TEA. Foram adotados os mesmos procedimentos metodológicos e analíticos usados nas determinações químicas dos solos antes do experimento.

Foram feitas análises de correlações simples (Pearson) entre a matéria seca e os teores e conteúdos de metais pesados da parte aérea e raízes das plantas apresentados por Andrade et al. (2009) com os teores trocáveis, não-trocáveis e pseudototais de metais pesados nos solos. Utilizou-se o Programa ASSISTAT desenvolvido no Centro de Tecnologia e Recursos Naturais da Universidade Federal de Campina Grande (PB).

\section{RESULTADOS E DISCUSSÃO}

\section{Teores pseudototais}

Os teores pseudototais de $\mathrm{Pb}, \mathrm{Cd}, \mathrm{Cr}, \mathrm{Cu}, \mathrm{Ni}$ e $\mathrm{Zn}$ variaram de 1,9 a 9.678,2 $\mathrm{mg} \mathrm{kg}^{-1}$ (Quadro 2). A digestão realizada pelo método U.S.EPA SW 846-3051A (água régia) não solubilizou totalmente a amostra de solo. Os elementos químicos na estrutura de minerais silicatados e óxidos refratários não são considerados móveis no meio ambiente e, portanto, excluídos de muitos mecanismos de transporte e poluição (USEPA, 2007). Portanto, considera-se que esse método extraiu o teor máximo potencialmente biodisponível dos metais pesados.

No solo 1 (referência), os teores de metais foram muito inferiores em relação aos demais solos (Quadro 2), confirmando as observações de campo (Quadro 1), em que essa área (mata nativa), localizada a montante da fábrica, não sofreu interferência direta das atividades de mineração. Contudo, os teores de $\mathrm{Pb}$ nesse solo foram superiores aos obtidos em estudos com solos não contaminados (teores naturais), usando o mesmo método de extração (Fadigas et al., 2002; Campos et al., 2003). O maior teor natural de $\mathrm{Pb}$ nos solos da região deste estudo é de origem litogênica, associado à grande ocorrência de veios de galena (Moraes, 1997; Silva, 1997).

Os teores elevados de $\mathrm{Pb}$ no solo 2 é decorrência de sua posição em meia encosta (Quadro 1), que favoreceu a adição de sedimentos ricos em rejeitos grosseiros e de coloração escura da metalurgia, vindos das partes mais altas. A montante desse ponto, há grande ocorrência de pilhas desses resíduos espalhados na superfície dos solos. 
Quadro 2. Teores pseudototais $\left(\mathrm{HNO}_{3} / \mathrm{HCl}\right)$, trocáveis (DTPA-TEA e $\left.\mathrm{Ca}\left(\mathrm{NO}_{3}\right)_{2}\right)$ e não trocáveis $\left(\mathrm{HNO}_{3}\right.$ 0,$5 ; 1,0$ e $4,0 \mathrm{~mol} \mathrm{~L}^{-1}$ ) de metais pesados nos $\operatorname{solos}^{(1)}$

\begin{tabular}{|c|c|c|c|c|c|c|}
\hline Solo & $\mathbf{P b}$ & Cd & $\mathrm{Cr}$ & $\mathrm{Cu}$ & $\mathrm{Ni}$ & $\mathrm{Zn}$ \\
\hline & \multicolumn{6}{|c|}{$-\mathrm{mg} \mathrm{kg}^{-1}$} \\
\hline & \multicolumn{6}{|c|}{$\mathrm{HNO}_{3} / \mathrm{HCl}$} \\
\hline 1 & 234,8 & 3,8 & 8,9 & 49,2 & 16,5 & 57,1 \\
\hline 2 & $4.504,9$ & 1,9 & 15,2 & 165,5 & 24,2 & 123,9 \\
\hline 3 & $9.678,2$ & 22,1 & 27,8 & 969,1 & 38,4 & 894,8 \\
\hline \multirow[t]{2}{*}{4} & $2.598,5$ & 2,1 & 18,0 & 175,9 & 22,6 & 87,5 \\
\hline & \multicolumn{6}{|c|}{ DTPA -TEA } \\
\hline 1 & 63,44 & nd & nd & 6,63 & 3,12 & 11,45 \\
\hline 2 & 977,08 & nd & nd & 16,81 & 1,46 & 1,44 \\
\hline 3 & 746,99 & nd & nd & 153,88 & 6,90 & 104,79 \\
\hline \multirow[t]{2}{*}{4} & 528,11 & nd & nd & 15,40 & 0,08 & 2,77 \\
\hline & \multicolumn{6}{|c|}{$\mathrm{Ca}\left(\mathrm{NO}_{3}\right)_{2}$} \\
\hline 1 & 0,49 & nd & nd & nd & 1,11 & nd \\
\hline 2 & 5,20 & nd & nd & nd & 0,25 & nd \\
\hline 3 & 426,67 & nd & nd & 1,13 & 7,57 & 157,14 \\
\hline \multirow[t]{2}{*}{4} & 1,39 & nd & & nd & 0,12 & nd \\
\hline & \multicolumn{6}{|c|}{$\mathrm{HNO}_{3} 0,5$} \\
\hline 1 & 271,6 & 2,5 & 1,2 & 5,1 & 4,1 & 27,0 \\
\hline 2 & $2.989,4$ & 1,2 & 0,7 & 24,3 & 0,8 & 15,0 \\
\hline 3 & $7.722,3$ & 21,1 & 1,8 & 626,2 & 19,0 & 602,9 \\
\hline \multirow[t]{2}{*}{4} & $1.165,0$ & 1,7 & 0,1 & 24,2 & 7,6 & 12,8 \\
\hline & \multicolumn{6}{|c|}{$\mathrm{HNO}_{3} 1,0$} \\
\hline 1 & 431,5 & 2,8 & 1,6 & 10,0 & 11,9 & 47,8 \\
\hline 2 & $3.962,9$ & 1,2 & 2,8 & 22,1 & 10,7 & 43,0 \\
\hline 3 & $9.155,2$ & 21,9 & 3,0 & 720,5 & 28,6 & 692,5 \\
\hline \multirow[t]{2}{*}{4} & $2.225,7$ & 1,4 & 1,8 & 37,7 & 9,5 & 41,5 \\
\hline & \multicolumn{6}{|c|}{$\mathrm{HNO}_{3} 4,0$} \\
\hline 1 & 439,5 & 2,8 & 17,0 & 48,6 & 18,3 & 65,0 \\
\hline 2 & $4.319,3$ & 1,4 & 18,5 & 113,2 & 20,5 & 85,8 \\
\hline 3 & $9.823,5$ & 22,0 & 19,4 & $1.023,8$ & 36,6 & 780,5 \\
\hline 4 & $2.453,0$ & 1,6 & 18,7 & 124,8 & 19,9 & 75,9 \\
\hline
\end{tabular}

Os maiores teores pseudototais foram encontrados no solo 3 (Quadro 2), localizado ao lado da fábrica, próximo de uma chaminé desativada. Esse solo, que apresentou os horizontes preservados e sem incorporação de resíduos sólidos, recebeu, por muitas décadas, grande deposição de materiais atmosféricos particulados, provenientes de poeiras liberadas na preparação (moagem) do minério e de fumaça liberada na fusão e metalurgia do $\mathrm{Pb}$. Com isso, esse material incorporado ao solo promoveu intensa poluição e aumentou significativamente os teores pseudototais de todos os metais pesados em relação ao solo de referência. Assim, os rejeitos e as escórias espalhados por toda área da Mina de Panelas de Brejaúvas, em Adrianópolis (PR), tiveram um papel agressivo ao meio ambiente, porém as emissões de materiais particulados pela preparação e beneficiamento do minério de $\mathrm{Pb}$ foram mais deletérias.

Conforme os Valores Orientadores para Solos Contaminados da CETESB para o Estado de São Paulo (CETESB, 2005), os teores de Pb nos solos 1, 2, 3 e 4;
Cd nos solos 1 e 3, e Cu e Zn no solo 3 (Quadro 2) estão acima do limite de intervenção agrícola $(\mathrm{Pb}$ $180 \mathrm{mg} \mathrm{kg}^{-1}$; Cd - 3 mg kg-1$; \mathrm{Cu}-200$ mg kg${ }^{-1} ; \mathrm{Zn}$ $450 \mathrm{mg} \mathrm{kg}^{-1}$ ). Para o $\mathrm{Pb}$, os teores nos solos 2,3 e 4 são muito superiores ao limite de intervenção industrial, que é bem menos restritivo ambientalmente $\left(900 \mathrm{mg} \mathrm{kg}^{-1}\right)$.

\section{Teores trocáveis}

Os maiores teores de metais pesados com DTPATEA (quelante orgânico polidentado) (Quadro 2) são devidos ao seu maior poder de extração em relação aos sais neutros, como o $\mathrm{Ca}\left(\mathrm{NO}_{3}\right)_{2}$ (Abreu et al., 1995, 1997; Milagres et al., 2007). Os solos sob influência direta das atividades de mineração (2, 3 e 4) também apresentaram elevados teores de $\mathrm{Pb}$ com DTPA-TEA, confirmando o grande risco de contaminação das águas. A íntima mistura de rejeitos sólidos no solo 2 favoreceu a solubilização do $\mathrm{Pb}$ e a passagem do elemento para formas mais livres (altos teores pelo DTPA-TEA) (Quadro 2). Verificou-se inversão nos teores de $\mathrm{Pb}$ DTPA-TEA entre os solos 2 e 3 em relação aos teores obtidos com água régia e com $\mathrm{Ca}\left(\mathrm{NO}_{3}\right)_{2}$.

$\mathrm{O}$ solo 3 também apresentou elevado teor de $\mathrm{Pb}$ trocável; o teor extraído com $\mathrm{Ca}\left(\mathrm{NO}_{3}\right)_{2}\left(426,67 \mathrm{mg} \mathrm{kg}^{-1}\right)$ é superior ao Valor de Intervenção Agrícola (180 $\mathrm{mg} \mathrm{kg}^{-1}$ ) estabelecido pela CETESB (2005), que adota a extração nítrico-clorídrica (água régia). Assim, considera-se que os solos de toda extensão próxima da fábrica devem merecer maior preocupação com a difusão de poluentes e contaminação ambiental.

\section{Teores não trocáveis}

Além da concentração 1,0 mol L-1 , utilizaram-se também soluções de $\mathrm{HNO}_{3}$ nas concentrações 0,5 e $4,0 \mathrm{~mol} \mathrm{~L}^{-1}$ (Quadro 2), visando estimar diferentes níveis de energia de ligação dos metais pesados aos coloides do solo. Os teores de $\mathrm{Pb}$ nos solos 2, 3 e 4 cresceram com o aumento da concentração do ácido. Comparando com os extratores DTPA-TEA e $\mathrm{Ca}\left(\mathrm{NO}_{3}\right)_{2}$, observa-se que as extrações nítricas apresentaram maior capacidade de extração de metais pesados, mesmo na concentração de $0,5 \mathrm{~mol} \mathrm{~L}^{-1}$. A solução de $\mathrm{HNO}_{3} 4,0 \mathrm{~mol} \mathrm{~L}^{-1}$ extraiu os metais pesados com energia semelhante à extração com água régia (Quadro 2).

No solo 3 , devido à alta labilidade das formas de $\mathrm{Pb}$ e $\mathrm{Zn}$ proveniente das emissões atmosféricas, a extração com $\mathrm{HNO}_{3}$ 0,5 mol L-1 representou $79 \%$ e $77 \%$, respectivamente, dos teores obtidos com a maior concentração desse ácido.

\section{Correlações entre os teores de metais pesados nos solos obtidos com diferentes extratores}

Não foram estabelecidas correlações para $\mathrm{Cd}$ e $\mathrm{Cr}$ pelos baixos teores trocáveis, não trocáveis e pseudototais desses elementos (Quadro 2). Com exceção da solução extratora DTPA-TEA para $\mathrm{Pb}$, os 
métodos $\mathrm{Ca}\left(\mathrm{NO}_{3}\right)_{2}, \mathrm{HNO}_{3}$ e água régia se correlacionaram muito bem entre si quanto à extração de metais pesados (Quadro 3). A menor correlação entre o DTPA-TEA e os métodos de natureza ácida é reflexo de princípios distintos de extração: complexação e ataque ácido. A complexação é mais eficiente para extrair metais associados à matéria orgânica e os métodos ácidos extraem com maior facilidade os metais adsorvidos e na rede cristalina de minerais (solubilização total ou parcial de minerais). Como já destacado, para o DTPA-TEA, em relação aos demais extratores, verificou-se inversão nos teores de $\mathrm{Pb}$ nos solos 2 e 3 (Quadro 2). No trabalho de Abreu et al. (1995), apesar da maior capacidade de extração de metais pesados pela solução Mehlich-3 em relação ao Mehlich-1, os coeficientes de correlação linear entre esses métodos foram significativos para $\mathrm{Pb}, \mathrm{Cd}, \mathrm{Cr}$ e Ni. Já as correlações entre teores de Pb por DTPA e as soluções de Mehlich- 1 e 3 foram menores e não significativas.

Os solos com maior teor de metais ligados fortemente aos coloides ou na estrutura de minerais também apresentaram maiores teores adsorvidos não especificamente - alta correlação entre $\mathrm{Ca}\left(\mathrm{NO}_{3}\right)_{2} \mathrm{e}$ extratores ácidos (Quadro 3) - ou seja, nos solos as formas desses poluentes se encontram em estreito equilíbrio.

Quadro 3. Correlações lineares simples entre os teores de $\mathrm{Pb}, \mathrm{Cu}, \mathrm{Zn}$ e $\mathrm{Ni}$ nos solos obtidos por diferentes extratores ${ }^{(1)}$

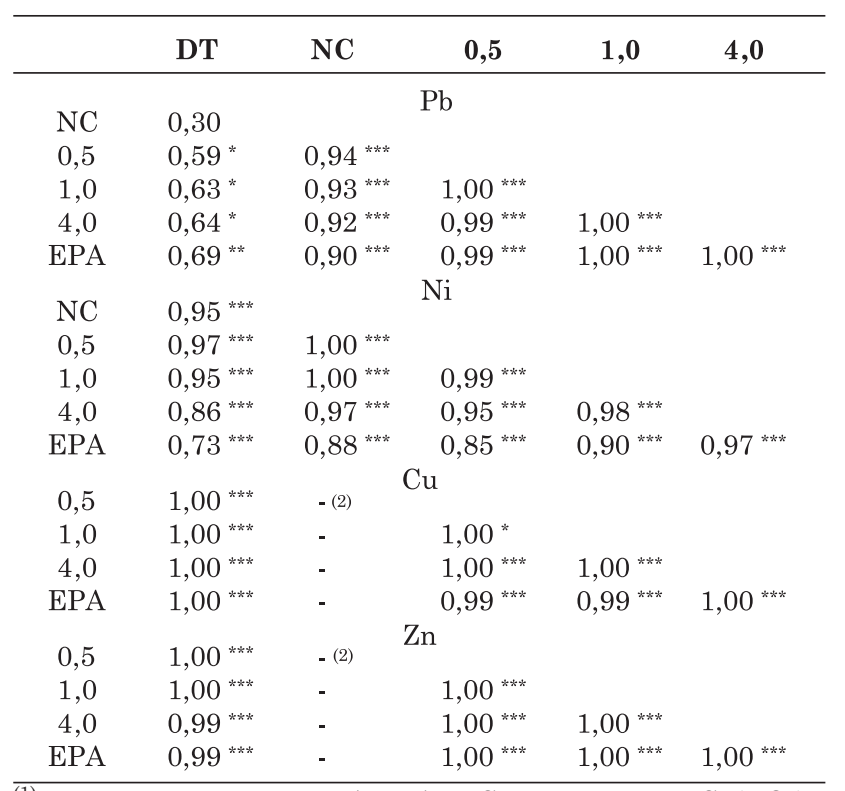

(1) DT: extração com DTPA-TEA; NC: extração com $\mathrm{Ca}\left(\mathrm{NO}_{3}\right)_{2}$ 0,5, 1,0 e 4,0: extração com soluções de $\mathrm{HNO}_{3}$ 0,5, 1,0 e 4,0 mol L-1, EPA: extração pelo método SW 846-3051A da U.S.EPA (água régia). * $* * \mathrm{e}^{* * *}$ : coeficientes de correlação $(\mathrm{r})$ significativos a a 5 a 1 , e a $0,1 \%$, respectivamente. ${ }^{(2)}$ Para $\mathrm{Cu}$ e $\mathrm{Zn}$, as correlações com $\mathrm{Ca}\left(\mathrm{NO}_{3}\right)_{2}$ não foram apresentadas pelos baixos teores (não detectado por ICP-AES - Quadro 2).
Teores de $\mathrm{Pb}$ no solo (DTPA-TEA e $\left.\mathrm{Ca}\left(\mathrm{NO}_{3}\right)_{2}\right)$ após experimento em casa de vegetação

No solo 1 (referência), observou-se discreta diminuição nos teores naturais de $\mathrm{Pb}$ extraídos com DTPA-TEA após o desenvolvimento das plantas (Quadros 2 e 4). Mesmo com a absorção pelas plantas, o cultivo induziu um aumento nos teores de $\mathrm{Pb}$ por $\mathrm{Ca}\left(\mathrm{NO}_{3}\right)_{2}$ no solo mais poluído (solo 3). Antes do cultivo, o teor era de $426,7 \mathrm{mg} \mathrm{kg}^{-1}$ e, após, variou de 518,8 a $530,0 \mathrm{mg} \mathrm{kg}^{-1}$ entre as espécies de plantas, ou seja, o cultivo de plantas estimulou a biodisponibilidade de $\mathrm{Pb}$ no solo.

O girassol promoveu maior redução nos teores de $\mathrm{Pb}$ DTPA-TEA no solo 2 após o cultivo em relação às demais espécies de plantas (diferença significativa $\mathrm{p}<0,05$ - Quadro 4).

\section{Métodos de extração química para estimar a disponibilidade de $\mathrm{Pb}$ e $\mathrm{Zn}$ para as plantas}

Devido aos baixos teores de $\mathrm{Cd}$, $\mathrm{Cr}$ e Ni nos solos (Quadro 2), trabalhou-se apenas com $\mathrm{Pb}$ e $\mathrm{Zn}$ para estabelecer os extratores mais eficientes para predição de suas disponibilidades para as plantas cultivadas.

Com exceção do extrator DTPA-TEA, os demais extratores apresentaram boas correlações com os teores de $\mathrm{Pb}$ nas raízes e parte aérea das plantas, considerando o conjunto das amostras ou as espécies separadamente (Quadro 5). O grande número de correlações altas e significativas é atribuído à relação estreita entre os próprios extratores (Quadro 3). A falta de ajuste para o DTPA foi devida à inversão nos teores de $\mathrm{Pb}$ entre os solos 2 e 3 (Quadro 2), em relação aos demais extratores, o que não foi acompanhado pela absorção das plantas; no solo 3 foram verificados maiores teores de $\mathrm{Pb}$ nas raízes e parte aérea das plantas (Andrade et al., 2009)

Quadro 4. Teores de $\mathrm{Pb}$ trocáveis extraídos por DTPA-TEA e por $\mathrm{Ca}\left(\mathrm{NO}_{3}\right)_{2}$ nos solos após experimento em casa de vegetação ${ }^{(1)}$

\begin{tabular}{crrr}
\hline \multirow{2}{*}{ Solo } & Girassol & Aveia-preta & Grama-batatais \\
\hline \multicolumn{4}{c}{ mg kg $^{-1}$} \\
\cline { 2 - 4 } & DTPA - TEA \\
1 & $47,06 \mathrm{dA}$ & $56,47 \mathrm{dA}$ & $52,45 \mathrm{dA}$ \\
2 & $930,46 \mathrm{aB}$ & $974,32 \mathrm{aA}$ & $989,75 \mathrm{aA}$ \\
3 & $795,39 \mathrm{bA}$ & $777,79 \mathrm{bA}$ & $771,61 \mathrm{bA}$ \\
4 & $571,84 \mathrm{cA}$ & $599,55 \mathrm{cA}$ & $572,83 \mathrm{cA}$ \\
& & $\mathrm{Ca}\left(\mathrm{NO}_{3}\right)_{2}$ & \\
1 & $0,87 \mathrm{~ns}$ & $0,26 \mathrm{~ns}$ & $0,42 \mathrm{~ns}$ \\
2 & $36,75 \mathrm{~ns}$ & $27,88 \mathrm{~ns}$ & $25,56 \mathrm{~ns}$ \\
3 & $530,03 \mathrm{~ns}$ & $518,77 \mathrm{~ns}$ & $528,20 \mathrm{~ns}$ \\
4 & $2,06 \mathrm{~ns}$ & $2,41 \mathrm{~ns}$ & $2,03 \mathrm{~ns}$
\end{tabular}

(1) Médias seguidas da mesma letra não diferem entre si pelo teste de Tukey a $5 \%$ : indicação em letras minúsculas nas colunas e letras maiúsculas nas linhas. ns: valor de F não significativo a $5 \%$. 
Quadro 5. Correlações lineares simples entre os teores de $\mathrm{Pb}$ e de $\mathrm{Zn}$ no solo obtidos por diferentes extratores e produção de matéria seca, teores e conteúdos nas raízes e partes aéreas das plantas ${ }^{(1)}$

\begin{tabular}{|c|c|c|c|c|c|c|c|c|}
\hline & MSR & MSPA & MST & $\mathrm{PbR}$ & $\mathrm{CPbR}$ & PbPA & $\mathrm{CPbPA}$ & CPb RA \\
\hline & \multicolumn{8}{|c|}{ Todas as espécies } \\
\hline $\mathrm{PbNC}$ & $-0,39$ & $-0,48$ & $-0,48$ & $0,82^{* * *}$ & $0,71^{* *}$ & $0,85^{* * *}$ & 0,09 & 0,61 * \\
\hline $\mathrm{PbDT}$ & $-0,41$ & $-0,49$ & $-0,49$ & 0,32 & $0,51^{\circ}$ & $0,50^{\circ}$ & 0,60 * & 0,63 * \\
\hline $\mathrm{Pb} 0,5$ & $-0,43$ & $-0,52^{\circ}$ & $-0,52^{\circ}$ & $0,80^{* *}$ & $0,78^{* *}$ & $0,88^{* * *}$ & 0,31 & $0,75^{* *}$ \\
\hline $\mathrm{Pb} 1$ & $-0,48$ & $-0,59^{*}$ & $-0,58^{*}$ & $0,78^{* *}$ & $0,77^{* *}$ & $0,88^{* * *}$ & 0,31 & 0,74 ** \\
\hline $\mathrm{Pb} 4$ & $-0,49$ & $-0,59^{*}$ & $-0,59^{*}$ & $0,78^{* *}$ & $0,77^{* *}$ & $0,88^{* * *}$ & 0,31 & 0,74 ** \\
\hline \multirow[t]{2}{*}{$\mathrm{PbEPA}$} & $-0,51^{\circ}$ & $-0,61^{*}$ & $-0,61^{*}$ & $0,77^{* *}$ & $0,77^{* *}$ & $0,88^{* * *}$ & 0,33 & $0,75^{* *}$ \\
\hline & \multicolumn{8}{|c|}{ Girassol } \\
\hline $\mathrm{PbNC}$ & $-0,48$ & $-0,48$ & $-0,48$ & $0,99^{* * *}$ & 0,75 & $0,99^{* * *}$ & $-0,21$ & 0,33 \\
\hline $\mathrm{PbDT}$ & $-0,58$ & $-0,51$ & $-0,52$ & 0,42 & 0,74 & 0,42 & 0,78 & 0,83 \\
\hline $\mathrm{Pb} \mathrm{0,5}$ & $-0,55$ & $-0,53$ & $-0,53$ & $0,98^{*}$ & $0,91^{\circ}$ & $0,98^{*}$ & 0,28 & 0,60 \\
\hline $\mathrm{Pb} 1$ & $-0,62$ & $-0,60$ & $-0,60$ & $0,96^{*}$ & 0,89 & $0,97^{*}$ & 0,29 & 0,60 \\
\hline $\mathrm{Pb} 4$ & $-0,62$ & $-0,60$ & $-0,61$ & $0,96^{*}$ & 0,90 & $0,97^{*}$ & 0,29 & 0,60 \\
\hline \multirow[t]{2}{*}{ PbEPA } & $-0,65$ & $-0,63$ & $-0,63$ & $0,95^{*}$ & 0,90 & $0,97^{*}$ & 0,32 & 0,62 \\
\hline & \multicolumn{8}{|c|}{ Aveia - preta } \\
\hline $\mathrm{PbNC}$ & $-0,55$ & $-0,54$ & $-0,55$ & $1,00^{* * *}$ & $0,63^{*}$ & $0,99 *$ & $-0,25$ & 0,42 \\
\hline $\mathrm{PbDT}$ & $-0,45$ & $-0,46$ & $-0,46$ & 0,33 & 0,80 & 0,44 & 0,69 & 0,84 \\
\hline $\mathrm{Pb} \mathrm{0,5}$ & $-0,56$ & $-0,56$ & $-0,56$ & $0,96^{*}$ & 0,83 & $0,98^{*}$ & 0,06 & 0,67 \\
\hline $\mathrm{Pb} 1$ & $-0,62$ & $-0,62$ & $-0,62$ & $0,94^{\circ}$ & 0,82 & $0,97^{*}$ & 0,06 & 0,67 \\
\hline $\mathrm{Pb} 4$ & $-0,62$ & $-0,63$ & $-0,63$ & $0,94^{\circ}$ & 0,82 & $0,97^{*}$ & 0,07 & 0,67 \\
\hline \multirow[t]{2}{*}{ PbEPA } & $-0,64$ & $-0,65$ & $-0,65$ & $0,92^{\circ}$ & 0,83 & 0,96 * & 0,10 & 0,69 \\
\hline & \multicolumn{8}{|c|}{ Grama-batatais } \\
\hline $\mathrm{PbNC}$ & $-0,42$ & $-0,45$ & $-0,44$ & $0,99^{* * *}$ & 0,97 * & 0,99 * & 0,88 & $0,96^{*}$ \\
\hline $\mathrm{PbDT}$ & $-0,55$ & $-0,59$ & $-0,58$ & 0,44 & 0,49 & 0,41 & 0,66 & 0,51 \\
\hline $\mathrm{Pb} 0,5$ & $-0,49$ & $-0,53$ & $-0,52$ & $0,98^{*}$ & $0,99^{* * *}$ & $0,97^{*}$ & $0,98^{*}$ & $0,99^{* * *}$ \\
\hline $\mathrm{Pb} 1$ & $-0,56$ & $-0,59$ & $-0,59$ & $0,96^{*}$ & $0,98^{*}$ & $0,96^{*}$ & 0,97 * & $0,98^{*}$ \\
\hline $\mathrm{Pb} 4$ & $-0,56$ & $-0,60$ & $-0,59$ & $0,96^{*}$ & $0,98^{*}$ & $0,96^{*}$ & $0,97^{*}$ & $0,98^{*}$ \\
\hline \multirow[t]{3}{*}{ PbEPA } & $-0,56$ & $-0,60$ & $-0,59$ & $0,96^{*}$ & $0,98^{*}$ & $0,96 *$ & $0,97^{*}$ & $0,98^{*}$ \\
\hline & MSR & MSPA & MST & $\mathrm{ZnR}$ & CZnR & $\mathrm{ZnPA}$ & CZnPA & CZnRA \\
\hline & \multicolumn{8}{|c|}{ Todas as espécies } \\
\hline ZnDT & $-0,34$ & $-0,42$ & $-0,42$ & $0,94^{* * *}$ & $0,63^{*}$ & $0,99^{* * *}$ & $0,52^{\circ}$ & $0,60^{*}$ \\
\hline Zn 0,5 & $-0,38$ & $-0,47$ & $-0,46$ & $0,81^{* * *}$ & $0,63^{*}$ & $1,00^{* * *}$ & $0,50^{\circ}$ & 0,59 * \\
\hline $\mathrm{Zn} 1$ & $-0,39$ & $-0,47$ & $-0,47$ & $0,95^{* * *}$ & 0,63 * & $1,00^{* * *}$ & 0,49 & $0,58^{*}$ \\
\hline Zn4 & $-0,40$ & $-0,49$ & $-0,49$ & $0,95^{* * *}$ & $0,62^{*}$ & $0,99^{* * *}$ & 0,48 & $0,57^{*}$ \\
\hline \multirow[t]{2}{*}{ ZnEPA } & $-0,47$ & $-0,41$ & $-0,43$ & $0,86^{* * *}$ & $-0,07$ & $0,66^{* *}$ & $-0,05$ & $-0,06$ \\
\hline & \multicolumn{8}{|c|}{ Girassol } \\
\hline ZnDT & $-0,41$ & $-0,42$ & $-0,42$ & 0,97 & $0,99^{* * *}$ & $1,00^{* * *}$ & 0,52 & 0,67 \\
\hline $\mathrm{Zn} 0,5$ & $-0,46$ & $-0,47$ & $-0,47$ & $1,00^{* * *}$ & $0,99^{*}$ & $1,00^{* * *}$ & 0,48 & 0,64 \\
\hline Zn1 & $-0,47$ & $-0,48$ & $-0,48$ & $1,00^{* * *}$ & $0,98^{*}$ & $1,00^{* * *}$ & 0,47 & 0,63 \\
\hline Zn4 & $-0,49$ & $-0,49$ & $-0,49$ & $1,00^{* * *}$ & $0,98^{*}$ & $1,00^{* * *}$ & 0,46 & 0,61 \\
\hline \multirow[t]{2}{*}{ Zn EPA } & $-0,50$ & $-0,51$ & $-0,51$ & $1,00^{* * *}$ & $0,98^{*}$ & $1,00^{* * *}$ & 0,44 & 0,60 \\
\hline & \multicolumn{8}{|c|}{ Aveia-preta } \\
\hline $\mathrm{ZnDT}$ & $-0,49$ & $-0,49$ & $-0,49$ & $0,99^{* * *}$ & 0,31 & $1,00^{* * *}$ & $-0,20$ & $-0,05$ \\
\hline Zn 0,5 & $-0,53$ & $-0,53$ & $-0,53$ & $1,00^{* * *}$ & 0,27 & $1,00^{* * *}$ & $-0,24$ & $-0,09$ \\
\hline $\mathrm{Zn} 1$ & $-0,54$ & $-0,54$ & $-0,54$ & $1,00^{* * *}$ & 0,26 & $1,00^{* * *}$ & $-0,26$ & $-0,10$ \\
\hline Zn4 & $-0,55$ & $-0,55$ & $-0,55$ & $1,00^{* * *}$ & 0,25 & $1,00^{* * *}$ & $-0,27$ & $-0,12$ \\
\hline \multirow[t]{2}{*}{ ZnEPA } & $-0,56$ & $-0,56$ & $-0,56$ & $1,00^{* * *}$ & 0,24 & $1,00^{* * *}$ & $-0,29$ & $-0,13$ \\
\hline & \multicolumn{8}{|c|}{ Grama-batatais } \\
\hline $\mathrm{ZnDT}$ & $-0,36$ & $-0,38$ & $-0,37$ & $1,00^{* * * *}$ & $1,00^{* * *}$ & $1,00^{* * *}$ & $0,96^{*}$ & $0,98^{*}$ \\
\hline $\mathrm{Zn} 0,5$ & $-0,41$ & $-0,43$ & $-0,42$ & $1,00^{* * *}$ & $1,00^{* * *}$ & $1,00^{* * *}$ & $0,94^{\circ}$ & $0,98^{*}$ \\
\hline $\mathrm{Zn} 1$ & $-0,42$ & $-0,44$ & $-0,43$ & $1,00^{* * *}$ & $1,00^{* * *}$ & $1,00^{* * *}$ & $0,94^{\circ}$ & $0,97^{*}$ \\
\hline Zn4 & $-0,43$ & $-0,46$ & $-0,45$ & $1,00^{* * *}$ & $0,99^{* * *}$ & $1,00^{* * *}$ & $0,93^{\circ}$ & $0,97^{*}$ \\
\hline ZnEPA & 0,16 & 0,15 & 0,15 & 0,85 & 0,87 & 0,84 & 0,86 & 0,87 \\
\hline
\end{tabular}

DT: extração com DTPA-TEA; NC: extração com $\mathrm{Ca}\left(\mathrm{NO}_{3}\right)_{2}$; 0,5, 1 e 4: extração com soluções de $\mathrm{HNO}_{3}$ 0,5, 1,0 e 4,0 mol L-1; EPA: extração pelo método SW 846-3051A da U.S.EPA (água régia); MSR: matéria seca de raiz, MSPA: matéria seca de parte aérea, MST: matéria seca total, $\mathrm{PbR}$ : teor de $\mathrm{Pb}$ na raiz, $\mathrm{CPbR}$ : conteúdo de $\mathrm{Pb}$ na raiz, $\mathrm{PbPA}$ : teor de $\mathrm{Pb}$ na parte aérea, $\mathrm{CPbPA}$ : conteúdo de $\mathrm{Pb}$ na parte aérea, CPbRA: conteúdo de $\mathrm{Pb}$ total na raiz e parte aérea. ${ }^{\circ},{ }^{*}, * * \mathrm{e} * * *$ : coeficientes de correlação (r) significativos a 10, 5, 1 e 0,1 \%, respectivamente. n para as correlações com todas as espécies $=12$, n para as correlações com cada espécie $=4$. 
As soluções de $\mathrm{Ca}\left(\mathrm{NO}_{3}\right)_{2}$ e $\mathrm{HNO}_{3} 0,5 \mathrm{~mol} \mathrm{~L}^{-1}$ apresentaram altos valores de coeficientes de correlação com a absorção de $\mathrm{Pb}$ pelas plantas, principalmente considerando-se as espécies separadas (Quadro 5). Contudo, deve-se descartar a extração com $\mathrm{Ca}\left(\mathrm{NO}_{3}\right)_{2}$, pois os altos valores de coeficientes de correlação foi devido apenas à formação de dois grupos distintos com amostras com teores próximos de zero (solos 1,2 e 4) e o solo 3 com altos teores de $\mathrm{Pb}$ (Figura 1). Já nas extrações nítricas e com água régia, verificou-se melhor distribuição dos pontos nas curvas de calibração. Um ponto favorável para a escolha do $\mathrm{HNO}_{3} 0,5 \mathrm{~mol} \mathrm{~L}^{-1}$ é a menor energia de extração em relação às demais extrações nítricas e água régia, o que disponibiliza quantidade de $\mathrm{Pb}$ mais próxima da absorção pela planta.

Mesmo com valores baixos, os coeficientes de correlações entre os teores de $\mathrm{Pb}$ no solo e a produção de matéria seca das plantas foram negativos (Quadro 5).

De forma similar ao $\mathrm{Pb}$, todos extratores apresentaram correlações significativas em relação à concentração de $\mathrm{Zn}$ nas raízes e parte aérea das plantas. As principais diferenças em relação ao $\mathrm{Pb}$ foram os maiores valores dos coeficientes de correlações para o extrator DTPA para Zn e correlações não significativas entre o Zn extraído por água régia (EPA) e os teores nas raízes e parte aérea da gramabatatais (Quadro 5); os teores de Zn advindos de formas mais estáveis não representaram satisfatoriamente a disponibilidade do elemento no solo para essa espécie.

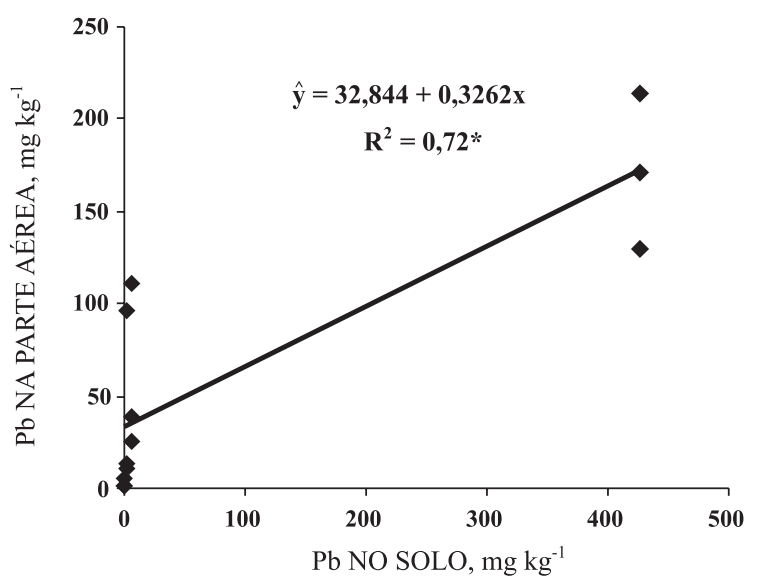

Figura 1. Regressão linear entre os teores de $\mathrm{Pb}$ dos solos extraídos por solução de $\mathrm{Ca}\left(\mathrm{NO}_{3}\right)_{2}$ $0,5 \mathrm{~mol} \mathrm{~L}^{-1}$ e teores na parte aérea das plantas.

\section{CONCLUSÕES}

1. A mineração e metalurgia de $\mathrm{Pb}$ promoveram grande poluição dos solos da área, com alto incremento nos teores pseudototais, trocáveis e não trocáveis de metais pesados.
2. Os extratores com princípio de solubilização (extratores ácidos - $\mathrm{HNO}_{3}$ 0,5; 1,0 e 4,0 mol L $\mathrm{L}^{-1}$ água régia) correlacionaram entre si quanto à extração de metais pesados nos solos. As extrações nítricas devem ser preferidas para se estabelecer a disponibilidade de $\mathrm{Pb}$ e Zn nos solos da área.

3. O extrator com princípio de quelação (DTPATEA pH 7,3) não foi eficiente na predição da disponibilidade desses metais para as plantas nas condições do deste estudo.

\section{LITERATURA CITADA}

ABREU, C.A.; ABREU, M.F.; RAIJ, B.van \& SANTOS, W.R. Comparação de métodos de análise para avaliar a disponibilidade de metais pesados em solos. R. Bras. Ci. Solo, 19:463-468, 1995.

ABREU, C.A.; ABREU, M.F.; HARADA, L.S. \& ANDRADE, J.C. The effects of the DTPA extraction conditions on the determination of micronutrients in Brazilian soils. Comm. Soil Sci. Plant Anal., 28:1-11, 1997.

ALLOWAY, B.J. Heavy metals in soils. Glasgow, Blackie Academic \& Professional, 1995. 368p.

ANDRADE, M.G.; MELO, V.F.; GABARDO, J.; SOUZA, L.C.P. \& REISSMANN, C.B. Metais pesados em solos de área de mineração e metalurgia de chumbo: I - Fitoextração. R. Bras. Ci. Solo, 33: 1829-1836, 2009.

BORGES, M.R. \& COUTINHO, E.L.M. Metais pesados do solo após aplicação de biossólidos. II - Disponibilidade. R. Bras. Ci. Solo, 28:557-568, 2004.

CAMPOS, M.L.; PIERANGELI, M.A.P.; GUILHERME, L.R.G.; MARQUES, J.J. \& CURI, N. Baseline concentration of heavy metals in Brazilian Latosols. Comm. Soil Sci. Plant Anal., 34:547-558, 2003.

CAMPOS, M.L.; SILVA, F.N.; FURTINI NETO, A.E.; GUILHERME, L.R.G.; MARQUES, J.J. \& ANTUNES, A.S. Determinação de cádmio, cobre, cromo, níquel, chumbo e zinco em fosfato de rocha. Pesq. Agropec. Bras., 40:361-367, 2005

CASSIANO, A.M. Fonte de contaminação por elementos-traço na bacia do rio Ribeira de Iguape (SP-PR): Estratégia para a remediação de uma área de disposição de rejeitos de mineração de Pb-Ag. São Carlos, Escola de Engenharia de São Carlos, Universidade de São Paulo, 2001. 159p. (Tese de Doutorado)

COMPANHIA DE TECNOLOGIA DE SANEAMENTO AMBIENTAL - CETESB. Decisão de diretoria $\mathrm{n}^{\circ} 195$ 2005 para valores orientadores para solos e águas subterrâneas no Estado de São Paulo. São Paulo, 2005.

FADIGAS, F.S.; AMARAL SOBRINHO, N.M.B.; MAZUR, N.; ANJOS, L.H.C. \& FREIXO, A.A. Concentrações naturais de metais pesados em algumas classes de solos brasileiros. Bragantia, 61:151-159, 2002.

HUNTER, A.H. Laboratory analysis of vegetal tissues samples. Raleigh, North Carolina University, 1975. 5p. 
KNUDSEN, D.; PETERSON, G.A. \& PRATT, P.F. Lithium, sodium and potassium. In: PAGE, A.L., ed. Methods of soil analysis. Chemical and microbiological properties. Madison, American Society of Agronomy, 1986. Part 2. p.225-246.

LINDSAY, W.L. \& NORVELL, W.A. Development of DTPA soil for zinc, iron, manganese and copper. Soil Sci. Soc. Am. J., 42:421-428, 1978.

MELO, E.E.C.; NASCIMENTO, C.W.A. \& SANTOS, A.C.Q. Solubilidade, fracionamento e fitoextração de metais pesados após aplicação de agentes quelantes. R. Bras. Ci. Solo, 30:1051-1060, 2006.

MILAGRES, J.J.M.; ALVAREZ V, V.H.; CANTARUTTI, R.B. \& NEVES, J.C.L. Determinação de $\mathrm{Fe}, \mathrm{Zn}, \mathrm{Cu}$ e $\mathrm{Mn}$ extraídos do solo por diferentes extratores e dosados por espectrofotometria de emissão ótica em plasma induzido e espectrofotometria de absorção atômica. R. Bras. Ci. Solo, 31:237-245, 2007.

MILLER, W.P.; MARTENS, D.C. \& ZELAZNY, L.W. Effect of sequence in extraction of trace metals from soils. Soil Sci. Soc. Am. J., 50:598-601, 1986.

MORAES, R.P. Transporte de chumbo e metais associados no Rio Ribeira de Iguape, São Paulo, Brasil. Campinas, Universidade Estadual de Campinas, 1997. 94p. (Tese de Mestrado)

NIEUWENHUIZE, J.; POLEY-VOS, C.H.; van der AKKER, A.H. \& van DELFT, W. Comparison of microwave and conventional extraction techniques for the determination of metals in soil, sediment and sludge samples by atomic spectrometry. Analyst, 116:347-351, 1991.
PIERANGELI, M.A.P.; GUILHERME, L.R.G.; CURI, N.; COSTA, E.T.S.; LIMA, J.M.; MARQUES, J.J.G.S.M. \& FIGUEIREDO, L.F.P. Comportamento sortivo, individual e competitivo, de metais pesados em Latossolos com mineralogia contrastante. R. Bras. Ci. Solo, 31:819$826,2007$.

RAURET, G. Extraction procedures for the determination of heavy metals in contaminated soil and sediment. Talanta, 46:449-455, 1998

SCHALSCHA, E.B.; MORALES, M.; AHUMADA, T.; SCHIRADO, T. \& PRATT, P.F. Fractionation of $\mathrm{Zn}, \mathrm{Cu}$, $\mathrm{Cr}$ and $\mathrm{Ni}$ in waste-water solids and in soil. Agrochimica, 24:361-368, 1980

SILVA, R.H.P. Geoquímica e impacto ambiental do arsênio no Vale do Ribeira (SP-PR). Campinas, Universidade Estadual de Campinas, 1997. 100p. (Tese de Mestrado)

SOON, Y.K. \& BATES, T.E. Chemical pools of Cd, Ni, and Zn in polluted soils and some preliminary indications of their availability to plants. J. Soil Sci., 33:477-488, 1982.

URE, A.M.; QUEVAUVILLER, P.; MUNTAU, H. \& GRIEPINK, B. Speciation of heavy metals in soils and sediments. An account of the improvement and harmonization of extraction techniques undertaken under the auspices of the BCR of the Commission of the European Communities. Intern. J. Environ. Anal. Chem., 51:135151, 1993.

U.S. ENVIRONMENTAL PROTECTION AGENCY - USEPA. Method 3051A - Microwave assisted acid digestion of sediments, sludges, soils, and oils. Washington, DC, 2007. 30p. Disponível em: <http://www.epa.gov/epawaste/ hazard/testmethods/sw846/pdfs/3051a.pdf> Revision 1, Acesso em: 07 March de 2007. 
